\title{
Optofluidic Refractive Index Sensor Based on Partial Reflection
}

\author{
Lei ZHANG ${ }^{1 *}$, Zhang ZHANG ${ }^{1}$, Yichuan $\mathrm{WANG}^{1}$, Meiying $\mathrm{YE}^{2}$, \\ Wei FANG $^{1 *}$, and Limin TONG ${ }^{1}$ \\ ${ }^{1}$ State Key Laboratory of Modern Optical Instrumentation, College of Optical Science and Engineering, Zhejiang \\ University, Hangzhou, 310027, China \\ ${ }^{2}$ College of Material, Chemistry and Chemical Engineering, Hangzhou Normal University, Hangzhou, 310036, China \\ *Corresponding author: Lei ZHANG and Wei FANG_E-mail: zhang_lei@zju.edu.cn and wfang08@zju.edu.cn
}

\begin{abstract}
We demonstrate a novel optofluidic refractive index (RI) sensor with high sensitivity and wide dynamic range based on partial reflection. Benefited from the divergent incident light and the output fibers with different tilting angles, we have achieved highly sensitive RI sensing in a wide range from 1.33 to 1.37 . To investigate the effectiveness of this sensor, we perform a measurement of RI with a resolution of $c a .5 .0 \times 10^{-5}$ refractive index unit (RIU) for ethylene glycol solutions. Also, we have measured a series of liquid solutions by using different output fibers, achieving a resolution of $c a .0 .52 \mathrm{mg} / \mathrm{mL}$ for cane surge. The optofluidic RI sensor takes advantage of the high sensitivity, wide dynamic range, small footprint, and low sample consumption, as well as the efficient fluidic sample delivery, making it useful for applications in the food industry.
\end{abstract}

Keywords: Optofluidic sensor; refractive index; partial reflection

Citation: Lei ZHANG, Zhang ZHANG, Yichuan WANG, Meiying YE, Wei FANG, and Limin TONG, "Optofluidic Refractive Index Sensor Based on Partial Reflection," Photonic Sensors, 2017, 7(2): 97-104.

\section{Introduction}

Miniaturized optofluidic devices offer great potential for realizing more functional and more compact optical systems through the fusion of microfluidics and optics $[1,2]$. The past decade has witnessed the realizations of optofluidic devices including tunable waveguides $[3,4]$, lenses $[5,6]$, switches $[7,8]$, apertures $[9,10]$, reconfigurable lasers $[11,12]$, interferometers $[13,14]$, gratings [15, 16], and sensitive sensors with fast response and low sample consumption [17-20], as well as demonstrations of their applications in chemical and biological analysis [21], energy [22], and photonics [23]. For many biological and chemical applications, the measurement of refractive index (RI) is extremely useful for detecting compounds that are: nonionic, transparent in the UV/vis range, or with no fluorescence, moreover, it has great potential for none invasive and label-free biosensing [24-26].

To date, a variety of optofluidic RI sensors have been reported by using integrated interferometers [27, 28], Fabry-Pérot cavities [29, 30], microring resonators [31, 32] gratings [33, 34], microstructured optical fibers [35, 36], and surface plasmon resonance [37,38]. Although the sensitivity of the aforementioned sensors can be as high as $10^{-6}$ RIU by measuring the wavelength shift, these sensors require expensive instruments, e.g., optical spectrum analyzers. Alternatively, one can design a

Received: 12 August 2016/ Revised: 22 September 2016

(C) The Author(s) 2017. This article is published with open access at Springerlink.com

DOI: $10.1007 / \mathrm{s} 13320-017-0369-5$

Article type: Regular 
highly sensitive RI sensor based on partial reflection or refraction by measuring the intensity of the reflected or transmitted light or both [39, 40]. It is well known that the reflectivity of liquid-solid interface can be approximated by combining Snell's law with the Fresnel equations of reflection. When the RI of the liquid increases to a value where total internal reflection (TIR) at the liquid-solid interface is no longer satisfied, the reflectivity will decrease sharply, leading to a strong increase of the transmitted light. This phenomenon provides an attractive feature for assembling tunable optofluidic device and RI sensors. For example, Lapsley and co-authors [39] reported a variable optical attenuator where the light attenuation was achieved by adjusting the RI of the liquid in the microfluidic channel and thus altering the reflectivity of the light at the sidewall of the microchannel. Recently, Weber and Vellekoop [40] reported an optofluidic sensor, which was consisted of one input fiber and two out fibers with fixed angles for guiding the incident light, reflected light, and refracted light, respectively. Also, integrated microlenses were used for collimating the divergent light. Note that an inherent disadvantage of this sensor is the narrow dynamic range, typically, 0.01 refractive index unit (RIU) for a fixed incident angle. Thus, one has to prepare many chips with different incident angles to determine samples within a wide range of RI.

In this work, we report an optofluidic RI sensor with one straight detection channel, two input fibers, and ten output fibers for RI sensing with high sensitivity and wide dynamic range. Compared with the existing counterparts that require microlenses for light collimation, our design takes advantage of the divergent incident light and the optimized position of the output fibers to realize high sensitive sensing in a dynamic range from 1.33 to 1.37 . In this case, we can measure the RI of most aqueous samples with high sensitivity by choosing an output fiber with a specific tilting angle. Since microlenses are removed from our design, the fabrication of the sensor becomes much simpler and cost-effective. To investigate the effectiveness of the RI sensor, we perform a measurement of ethylene glycol (EG) solutions with a RI range from 1.33 to 1.37 , achieving a resolution of ca. $5.0 \times 10^{-5}$ RIU. Furthermore, we present measurements of liquid concentrations with a resolution of $c a .0 .52 \mathrm{mg} / \mathrm{mL}$ for cane surge. The optofluidic RI sensor shown here may provide a compact and versatile sensing platform for sensitive and fast detection of low-volume samples.

\section{Experimental section}

\subsection{Reagents and instruments}

All reagents and standards were of analytical grade and purchased from Sinopharm Chemical Reagent (Shanghai, China) unless otherwise stated. SU-8 photoresist and SU-8 developer were purchased from MicroChem Corp. (Newton, MA, USA). Polydimethylsiloxane (PDMS) (Sylgard 184) was purchased from Dow Corning (Midland, MI, USA). Ultrapure water (Siemens Labostar2-UV) was used throughout. Sample solutions were prepared before use. Optical micrographs were obtained using a charge coupled device (CCD) camera (DS-Fi1, Nikon, Japan) mounted on a microscope (Eclipse 90i, Nikon, Japan).

\subsection{Fabrication of the optofluidic device}

Figure 1 shows an optical image of a PDMS microfluidic chip for multi-fiber RI sensing, in which it contains one detection channel, two fiber channels with a tilting angle of $71^{\circ}$, and ten output fibers channels with tilting angles range from $67^{\circ}$ to $76^{\circ}$, respectively. The tilting angle of $71^{\circ}$ was calculated by using Snell's law [40], and the tilting angles of the output fibers were defined according to the divergent incident light. The microfluidic chip was fabricated by using standard soft lithography process [41]. Briefly, uncured PDMS was poured onto a SU- 8 master, followed by curing at $80{ }^{\circ} \mathrm{C}$ for $20 \mathrm{~min}$. The cured PDMS slab was then peeled from the SU-8 master, and punched holes at the ends of 
the detection channel and the PDMS channels to introduce sample and uncured PDMS, respectively. The PDMS slab was bonded with a clean glass slide by using a plasma cleaner (PDC-32G-2, Harrick, USA). Typically, the detection channel was $2 \mathrm{~cm}$ in length, $125 \mu \mathrm{m}$ in width, and $150 \mu \mathrm{m}$ in depth with a rectangular cross section. In this case, optical fibers could be inserted into the fiber channels and well aligned by fiber channels. To remove the scattering light, the gap between the fiber and fiber channel was sealed by introducing uncured PDMS from the PDMS inlets, followed by curing at $80{ }^{\circ} \mathrm{C}$ for $20 \mathrm{~min}$.

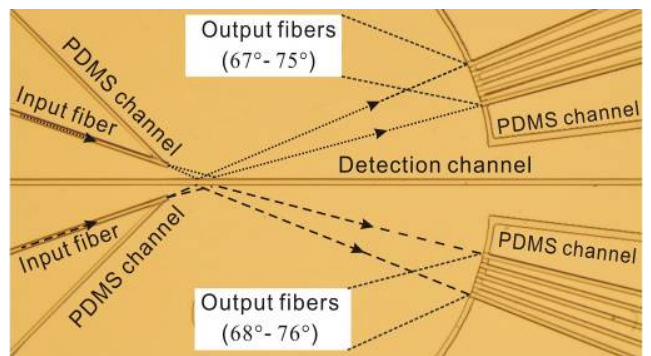

Fig. 1 Optical image of a microfluidic chip for multi-fiber RI sensing, which contains one detection channel, two input fibers channels, four PDMS channels, and ten output fiber channels. The dot and dash lines indicate the divergent incident light and reflected light, respectively.

\subsection{Procedures}

In the experiment, $5 \mu \mathrm{L}$ of sample solution was added into the sample inlet and was introduced into the detection channel by negative pressure generated by a syringe. After each measurement, the channel was flushed with ultrapure water. A $473 \mathrm{~nm}$ laser and a broadband light from a tungsten halide lamp (PHILIPS 7748XHP) were coupled into input fibers and served as incident light for visualization and RI measurements, respectively. The reflected light was collected by one of the ten output fibers and recorded by a spectrometer (Maya2000 Pro, Ocean optics, Dunedin, FL, USA).

\section{Working principle}

Figure 2(a) schematically shows a typical structure for illustrating the operating mechanism of the RI sensor, where $\alpha$ and $\beta$ denote the tilting angles of the input and output fibers, respectively, and $d$ denotes the distance between the end face of an output fiber and the reflection zone.

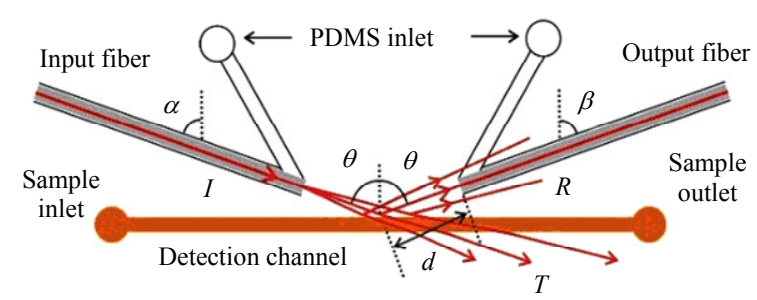

(a)

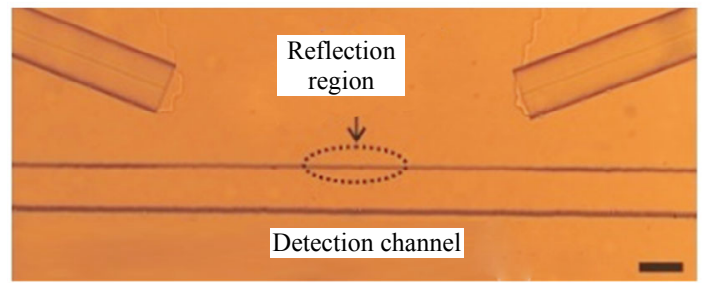

(b)

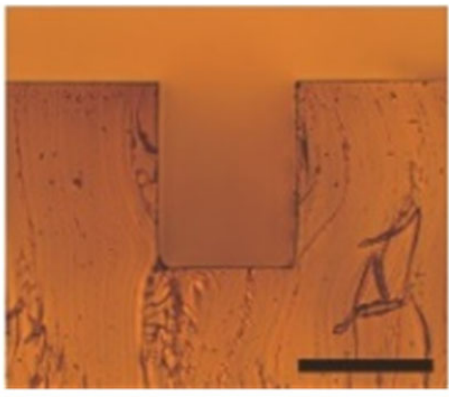

(c)

Fig. 2 Illustration of a microfluidic RI sensor: (a) a schematic illustration of a microfluidic RI sensor with one pair of fibers for optimizing the tilting angles of the input fiber and the distance between the end face of the output fiber to the reflection zone and (b) optical micrograph of a microfluidic RI sensor with $\alpha=\beta=71^{\circ}$, and $d=0.6 \mathrm{~mm}$, and (c) cross section of the detection channel. Scale bars: $125 \mu \mathrm{m}$.

Figure 2(b) shows an optical micrograph of an optofluidic RI sensor with $\alpha=\beta=71^{\circ}$ and $d=0.6 \mathrm{~mm}$. As shown in Fig. 2(c), the vertical sidewalls of the detection channel serve as reflective interfaces. When the liquid sample is introduced into the detection channel, a RI mismatch between PDMS and the liquid sample in the detection channel will cause the incident optical beam $(I)$ to be reflected and refracted at the PDMS-liquid interface, resulting in a reflected beam $(R)$ and a transmitted beam $(T)$. The angle of $R(\theta)$ is the same as the angle of $I$, allowing the reflected beam to be captured by the 
output optical fiber. The intensity of the reflected light is a function of RI of the liquid sample, and thus, we can determine the concentration of solutes by measuring the intensity of the reflected light. Note that we did not fabricate microlenses to collimate incident light, it offers a possibility to arrange several output fibers with different tilting angles to record the divergent reflected beam. In this case, each output fiber corresponds to an angle of reflection, providing an effective method to achieve a wide dynamic range. On the other hand, the insertion loss of the device is relatively greater than its counterparts with microlenses, and approximately 5\% incident light could be collected by the output fiber for the microfluidic chip as shown in Fig. 2(b).

\section{Results and discussion}

\subsection{Visualization of the reflected and refracted beam}

To visualize the reflected and refracted beams, a 473-nm-wavelength laser was used as incident light and $0.01 \mathrm{mM}$ fluorescein solutions with different RIs were used to visualize the refracted light. Figure 3 shows four typical images of an optofluidic RI sensor $\left(\alpha=\beta=71^{\circ}\right)$ with different fluids in the detection channel. When air $(n=1)$ was in the detection channel, TIR occurred at the PDMS-air interface, and the divergent reflected light could be clearly seen in Fig. 3(a). When the tilting angle of the input fiber was $71^{\circ}$, the estimated angle of reflection was from $67^{\circ}$ to $76^{\circ}$. When fluorescein solutions were injected into the detection channel, the fluorescence intensity increased obviously with an increase in RI from 1.33, 1.36, to 1.42 [Figs. 3(b)-(d)], indicating an increase in the refracted light intensity and a decrease in the reflected one. When the fluorescein solution with a RI of 1.42 was in the detection channel, the sidewall of the detection channel could hardly be identified, and the incident beam transmitted through the detection channel without obvious refraction and reflection [Fig. 3(d)].

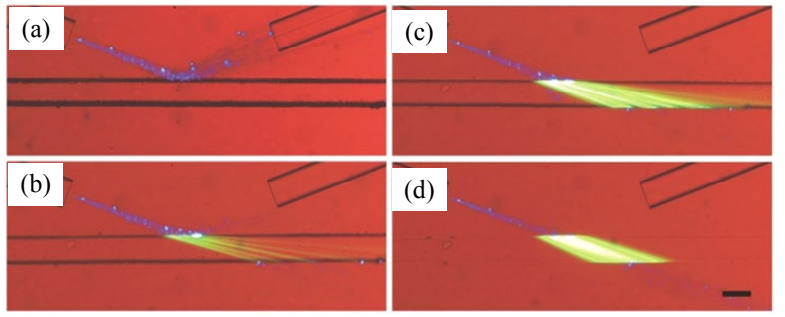

Fig. 3 Optical images of reflected and refracted light with different fluids within the detection channel: (a) air $(n=1)$ and (b)-(d) $0.01 \mathrm{mM}$ fluorescein was introduced into the detection channel as an indicator of the refracted light. The RI of the solutions were $1.33,1.36$, and 1.42 , respectively. Scale bar: $125 \mu \mathrm{m}$.

\subsection{Theoretical calculation}

To achieve a fundamental understanding of the sensor's RI response, theoretical analysis was conducted using Fresnel equations. We calculated the reflection coefficients $r_{s}$ and $r_{p}$ for s- and ppolarizations by (1) and (2), respectively, with the total reflectivity calculated by (3)

$$
\begin{gathered}
r_{s}=\frac{n_{1} \cos \theta-n_{2} \sqrt{1-\left(\frac{n_{1}}{n_{2}} \sin \theta\right)^{2}}}{n_{1} \cos \theta+n_{2} \sqrt{1-\left(\frac{n_{1}}{n_{2}} \sin \theta\right)^{2}}} \\
r_{p}=\frac{n_{2} \cos \theta-n_{1} \sqrt{1-\left(\frac{n_{1}}{n_{2}} \sin \theta\right)^{2}}}{n_{2} \cos \theta+n_{1} \sqrt{1-\left(\frac{n_{1}}{n_{2}} \sin \theta\right)^{2}}} \\
\bar{R}=\frac{\left|r_{s}\right|^{2}+\left|r_{p}\right|^{2}}{2}
\end{gathered}
$$

where $n_{1}=1.41$ is the RI of PDMS, and $n_{2}$ is the RI of the liquid in the detection channel. Thus, the reflectivity is a function of $n_{2}$. Figure 4(a) shows a theoretical analysis of the reflected light intensity of an RI sensor with different angles of reflection. For a fixed angle $\theta$, we found that the intensity kept as unity for small $n_{2}$ because of the TIR at the PDMS-liquid interface. When $n_{2}$ increased to a value where TIR was no longer satisfied, a very sharp decrease in reflectivity was observed with very small change in $n_{2}$. Based on theoretical prediction, the slope can be infinity right at the critical point, thus a very sensitive RI sensor could be realized. For example, when $n_{2}$ changed 0.001 
RIU, the maximum change in the intensity could be $30 \%$, however, the dynamic range of the sensor was ca. $0.01 \mathrm{RIU}$, which was relatively narrow for real application. As shown in Fig. 4(a), when $\theta$ increases from $69^{\circ}$ to $74^{\circ}$, the critical RI of $n_{2}$ shifts from 1.33 to 1.37 . Thus, we could achieve a wide dynamic range by choosing an output fiber with different $\theta$.

\subsection{Effect of $d$ on the measurement of RI}

To optimize $d$ of the RI sensor, we fabricated a series of microfluidic chips with one input fiber and one output fiber for characterization. Owing to the large aperture and core diameter, a multi-mode fiber could collect light from a wide angle, and thus smearing out the sharp decrease of the light coming from a single collection angle as indicated by the theoretical calculations [Fig. 4(a)]. In this work, we adopted SMFs with a core diameter around $10 \mu \mathrm{m}$ as input and output optical fibers, thus, the reflection light within a narrow range of angle could be collected by the output fiber. We found that $d$ had a great impact on the performance of the sensor. Figures 4(b) and 4(c) show normalized intensity of the light collected by the output fiber with different

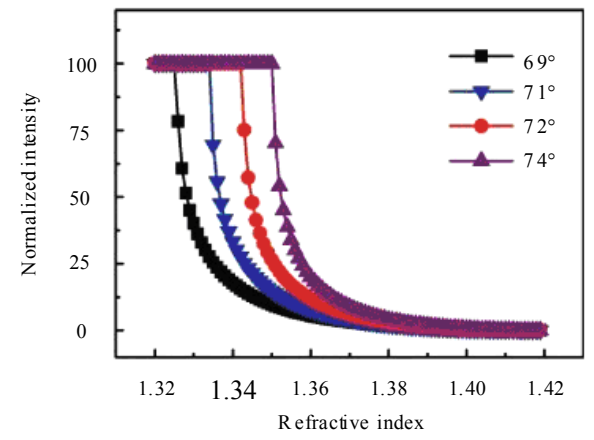

(a)

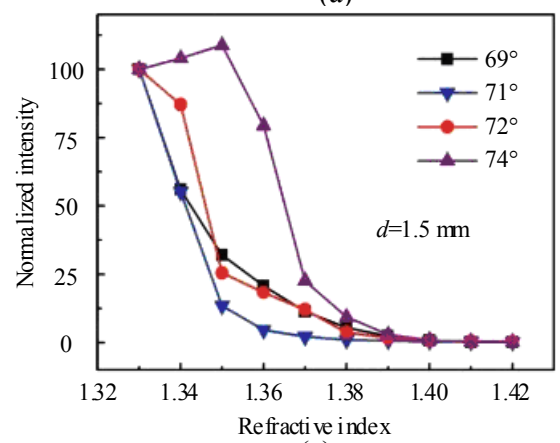

(c) tilting angles as a function of RI for $d$ of $0.6 \mathrm{~mm}$, and $1.5 \mathrm{~mm}$, respectively. The results did not match with the theoretical analysis [Fig. 4(a)], especially, the response curves for $\beta=69^{\circ}$ and $\beta=71^{\circ}$ can be hardly resolved in the RI range of $1.33-1.34$. This mismatch could be attributed to the output fiber collecting the light with different angles of reflection. Increasing $d$ is an effective method to narrow the range of the angle of reflection. When we increased $d$ to $5 \mathrm{~mm}$, the flare angle of the reflected light that could be collected by a SMF with a core diameter of $10 \mu \mathrm{m}$ decreased dramatically. To obtain the estimated flare angle of the reflected light, we considered the reflection area as a point, thus, the sides of the isosceles triangle were $5 \mathrm{~mm}$ and $10 \mu \mathrm{m}$, respectively, resulting in a flare angle of approximately $0.1^{\circ}$. As shown in Fig. 4(d), all of the four response curves for the different tilting angles are well separated, and the slopes after the critical points become steeper, which matched well with the theoretical calculations [Fig. 4(a)]. It is worth mentioning that we can arrange more output fiber on one side the detection to collect reflected light with different angles when $d$ is $5 \mathrm{~mm}$.

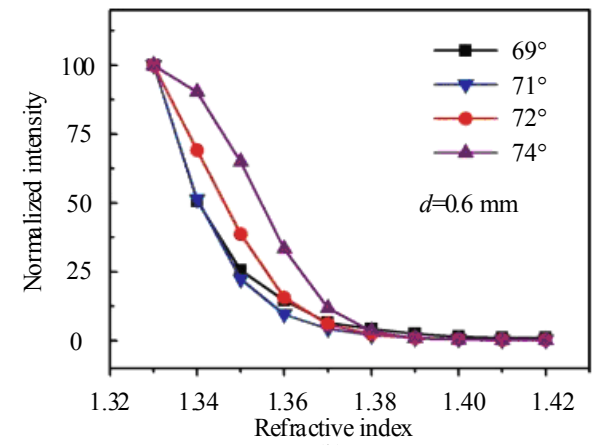

(b)

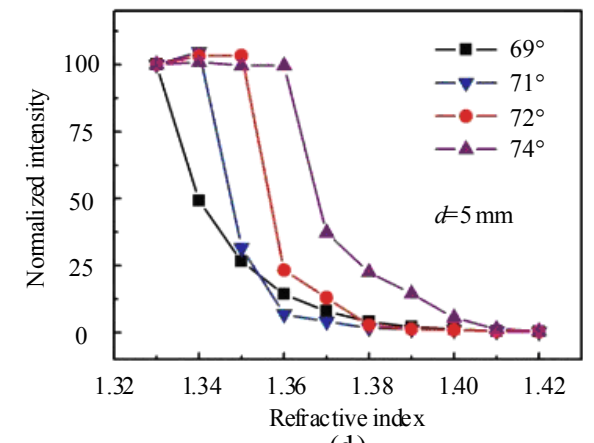

(d)

Fig. 4 Reflection response of the RI sensor for different $\theta$. (a) theoretical analysis of the reflection response of the RI sensor for different $\theta$ and (b)-(d) normalized intensity of the reflected light collected by four output fibers with reflected angles of $69^{\circ}, 71^{\circ}, 72^{\circ}$, and $74^{\circ}$, respectively, except for the variations to the distance between the endface of an output fiber and the reflection zone as indicated in the figures. 


\subsection{Performance of the multi-fiber RI sensor}

Figure 5 shows four typical curves recorded by the output fibers with a tilting angle of $69^{\circ}, 71^{\circ}, 72^{\circ}$, and $74^{\circ}$, respectively, which covers a RI range from 1.33 to 1.37 . The result confirmed that the dynamic range could be extended by using different output fibers for sensing. When RI increased from 1.35 to 1.36, the normalized intensity for the output fiber with a tilting angle of $72^{\circ}$ decreased from 103 to 23 . Because the stability of the light source was measured with an outstanding reproducibility of $0.13 \% \mathrm{RSD}$, the RI resolution could be estimated as $5.0 \times 10^{-5}$ RIU based on 3 times the standard deviation of the light source. Note that the signals collected by the output fibers with a tilting angle less than $68^{\circ}$ or greater than $74^{\circ}$ were too weak for sensing owing to the Gaussian intensity distribution of the divergent incident beam.

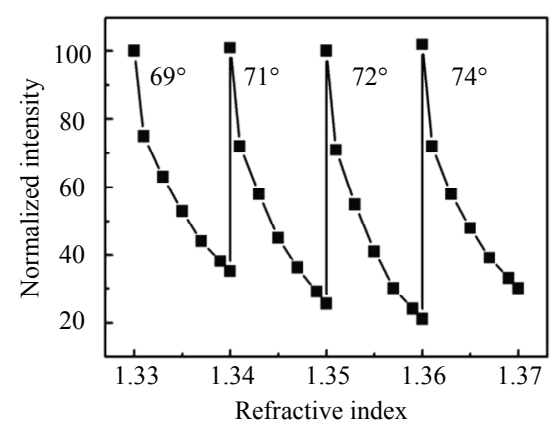

Fig. 5 Normalized intensity of the reflected light collected by four output fibers with tilting angles of $69^{\circ}, 71^{\circ}, 72^{\circ}$, and $74^{\circ}$, respectively.

Table 1 Measurement results of the concentration of cane surge obtained by using three output fibers with different tilting angles.

\begin{tabular}{ccccc}
\hline $\begin{array}{c}\text { Referenced } \\
\text { concentration } \\
(\mathrm{g} / \mathrm{mL})\end{array}$ & $\begin{array}{c}\text { Referenced } \\
\mathrm{RI}\end{array}$ & $\begin{array}{c}\text { Experimental } \\
\text { value } \\
(\mathrm{g} / \mathrm{mL})\end{array}$ & $\begin{array}{c}\text { Tilting } \\
\text { Angle }\end{array}$ & $\begin{array}{c}\mathrm{RE} \\
(\%)\end{array}$ \\
\hline 0.100 & 1.3459 & 0.105 & $71^{\circ}$ & 5.0 \\
0.150 & 1.3525 & 0.154 & $72^{\circ}$ & 2.7 \\
0.200 & 1.3586 & 0.192 & $72^{\circ}$ & -4.0 \\
0.250 & 1.3642 & 0.246 & $74^{\circ}$ & -1.6 \\
0.300 & 1.3692 & 0.307 & $74^{\circ}$ & 2.3 \\
\hline
\end{tabular}

To further evaluate the performance of the RI sensor, aqueous cane surge solutions with different concentrations $(0.1 \mathrm{~g} / \mathrm{mL}-0.3 \mathrm{~g} / \mathrm{mL})$ were prepared and analyzed. Because the RI of the cane surge solution is in a range from 1.34 to 1.37 , we adopted three output fibers with tilting angle of $71^{\circ}, 72^{\circ}$, and $74^{\circ}$ for collecting the signals, respectively. Table 1 gives obtained values and the relative errors (RE) for the sensor, showing a wide dynamic range and high accuracy with an estimated resolution of $0.52 \mathrm{mg} / \mathrm{mL}$.

\section{Conclusions}

In summary, we have developed a sensitive, robust, and inexpensive optofluidic RI sensor. We have realized highly sensitive RI sensing in a wide dynamic range of $1.33-1.37$ with a RI resolution of $5.0 \times 10^{-5}$ RIU by choosing an output fiber with different tilting angles for signal recording. Also, we have measured a series of liquid solutions by using different output fibers, achieving a resolution of $c a$. $0.52 \mathrm{mg} / \mathrm{mL}$ for cane surge. To further enhance the sensing performance, one might use a higher resolution lithography instrument to fabricate a SU-8 master with a smoother sidewall. We believe this optofluidic RI sensor can be used for real-time, low-cost, and multifunctional measurements in a wide range of applications.

\section{Acknowledgment}

This work has been supported in part by National Basic Research Program of China (Nos. 2013CB328703 and 2014CB921303), and National Natural Science Foundation of China (61275217, 21407039).

Open Access This article is distributed under the terms of the Creative Commons Attribution 4.0 International License (http://creativecommons.org/ licenses/by/4.0/), which permits unrestricted use, distribution, and reproduction in any medium, provided you give appropriate credit to the original author(s) and the source, provide a link to the Creative Commons license, and indicate if changes were made.

\section{References}

[1] D. Psaltis, S. R. Quake, and C. H. Yang, "Insight review: developing optofluidic technology through the fusion of microfluidics and optics," Nature, 2006, 442(7101): 381-386. 
[2] C. Monat, P. Domachuk, and B. J. Eggleton, "Integrated optofluidics: a new river of light," Nature Photonics, 2007, 1(2): 106-114.

[3] D. B. Wolfe, R. S. Conroy, P. Garstecki, B. T. Mayers, M. A. Fischbach, K. E. Paul, et al., "Dynamic control of liquid-core/liquid-cladding optical waveguides," Proceedings of the National Academy of Sciences, 2004, 101(34): 12434-12438.

[4] B. T. Mayers, D. V. Vezenov, V. I. Vullev, and G. M. Whitesides, "Arrays and cascades of fluorescent liquid-liquid waveguides: broadband light sources for spectroscopy in microchannels," Analytical Chemistry, 2005, 77(5): 1310-1316.

[5] X. L. Mao, J. R. Waldeisen, B. K. Juluri, and T. J. Huang, "Hydrodynamically tunable optofluidic cylindrical microlens," Lab on a Chip, 2007, 7(10): 1303-1308.

[6] P. Fei, Z. He, C. Zheng, T. Chen, Y. Men, and Y. Huang, "Discretely tunable optofluidic compound microlenses," Lab on a Chip, 2011, 11(17): 2835-2841.

[7] A. Groisman, S. Zamek, K. Campbell, L. Pang, U. Levy, and Y. Fainman, "Optofluidic $1 \times 4$ switch," Optics Express, 2008, 16(18): 13499-13508.

[8] W. Z. Song and D. Psaltis, "Electrically tunable optofluidic light switch for reconfigurable solar lighting," Lab on a Chip, 2013, 13(14): 2708-2713.

[9] Y. Hongbin, Z. Guangya, C. F. Siong, and L. Feiwen, "Optofluidic variable aperture," Optics Letters, 2008, 33(6): 548-550.

[10] C. L. Song, N. T. Nguyen, A. K. Asundi, and C. L. N. Low, "Tunable optofluidic aperture configured by a liquid-core/liquid-cladding structure," Optics Letters, 2011, 36(10): 1767-1769.

[11] Z. Li, Z. Zhang, T. Emery, A. Scherer, and D. Psaltis, "Single mode optofluidic distributed feedback dye laser," Optics Express, 2006, 14(2): 696-701.

[12] M. Aas, A. Jonas, A. Kiraz, O. Brzobohaty, J. Jezek, Z. Pilat, and P. Zemanek, "Spectral tuning of lasing emission from optofluidic droplet microlasers using optical stretching," Optics Express, 2013, 21(18): 21380-21394.

[13] C. Grillet, P. Domachuk, V. Ta'eed, E. Mägi, J. Bolger, B. Eggleton, et al., "Compact tunable microfluidic interferometer," Optics Express, 2004, 12(22): 5440-5447.

[14] Y. Zou, Z. Hen, X. Hen, Z. Di, and X. Chen, “An integrated tunable interferometer controlled by liquid diffusion in polydimethylsiloxane," Optics Express, 2012, 20(17): 18931-18936.

[15] M. Hashimoto, B. Mayers, P. Garstecki, and G. M. Whitesides, "Flowing lattices of bubbles as tunable, self-assembled diffraction gratings," Small, 2006, 2(11): 1292-1298.
[16] J. Q. Yu, Y. Yang, A. Q. Liu, L. K. Chin, and X. M. Zhang, "Microfluidic droplet grating for reconfigurable optical diffraction," Optics Letters, 2010, 35(11): 1890-1892.

[17] P. Liu, H. Huang, T. Cao, X. Liu, Z. Qi, Z. Tang, et al., "An ultra-low detection-limit optofluidic biosensor with integrated dual-channel Fabry-Pérot cavity," Applied Physics Letters, 2013, 102(16): 163701-1-163701-4.

[18] A. A. P. Trichet, J. Foster, N. E. Omori, D. James, P. R. Dolan, G. M. Hughes, et al., "Open-access optical microcavities for lab-on-a-chip refractive index sensing," Lab on a Chip, 2014, 14(21): 4244-4249.

[19] Z. Xu, K. Han, I. Khan, X. Wang, and G. L. Liu, "Liquid refractive index sensing independent of opacity using an optofluidic diffraction sensor," Optics Letters, 2014, 39(20): 6082-6085.

[20]C. Wu, M. L. V. Tse, Z. Liu, B. O. Guan, A. P. Zhang, C. Lu, et al., "In-line microfluidic integration of photonic crystal fibers as a highly sensitive refractometer," Analyst, 2014, 139(21): 5422-5429.

[21] X. D. Fan and I. M. White, "Optofluidic microsystems for chemical and biological analysis," Nature Photonics, 2011, 5(10): 591-297.

[22] D. Erickson, D. Sinton, and D. Psaltis, "Optofluidics for energy applications," Nature Photonics, 2011, 5(10): 583-590.

[23] L. Pang, H. M. Chen, L. M. Freeman, and Y. Fainman, "Optofluidic devices and applications in photonics, sensing and imaging," Lab on a Chip, 2012, 12(19): 3543-3551.

[24] P. J. Viskari and J. P. Landers, "Unconventional detection methods for microfluidic devices," Electrophoresis, 2006, 27(9): 1797-1810.

[25] Y. Wang, S. Meng, Y. Liang, L. Li, and W. Peng, "Fiber-optic surface plasmon resonance sensor with multi-alternating metal layers for biological measurement," Photonic Sensors, 2013, 3(3): 202-207.

[26] J. Zhu, L. Qin, S. Song, J. Zhong, and S. Lin, "Design of a surface plasmon resonance sensor based on grating connection," Photonic Sensors, 2015, 5(2): 159-165.

[27]L. K. Chin, A. Q. Liu, Y. C. Soh, C. S. Lim, and C. L. Lin, "A reconfigurable optofluidic Michelson interferometer using tunable droplet grating," Lab on a Chip, 2010, 10(8): 1072-1078.

[28] M. I. Lapsley, I. K. Chiang, Y. B. Zheng, X. Y. Ding, X. L. Mao, and T. J. Huang, "A single-layer, planar, optofluidic Mach-Zehnder interferometer for label-free detection," Lab on a Chip, 2011, 11(10): 1795-1800.

[29] W. Z. Song, X. M. Zhang, A. Q. Liu, C. S. Lim, P. H. Yap, and H. M. M. Hosseini, "Refractive index 
measurement of single living cells using on-chip Fabry-Pérot cavity," Applied Physics Letters, 2006, 89(20): 203901-1-203901-3.

[30] Y. Guo, H. Li, K. Reddy, H. S. Shelar, V. R. Nittoor, and $\mathrm{X}$. Fan, "Optofluidic Fabry-Pérot cavity biosensor with integrated flow-through micro-/ nanochannels," Applied Physics Letters, 2011, 98(4): 041104-1-041104-3.

[31] L. Q. Ren, X. Wu, M. Li, X. W. Zhang, L. Y. Liu, and $\mathrm{L}$. $\mathrm{Xu}$, "Ultrasensitive label-free coupled optofluidic ring laser sensor," Optics Letters, 2012, 37(18): 3873-3875.

[32] M. Li, X. Wu, L. Y. Liu, X. D. Fan, and L. Xu, "Self-Referencing optofluidic ring resonator sensor for highly sensitive biomolecular detection," Analytical Chemistry, 2013, 85(19): 9328-9332.

[33] W. Liang, Y. Y. Huang, Y. Xu, R. K. Lee, and A. Yariv, "Highly sensitive fiber Bragg grating refractive index sensors," Applied Physics Letters, 2005, 86(15): 151122-1-151122-3.

[34] F. Xu, G. Brambilla, and Y. Q. Lu, "A microfluidic refractometric sensor based on gratings in optical fibre microwires," Optics Express, 2009, 17(23): 20866-20871

[35] J. Wu, D. Day, and M. Gu, "A microfluidic refractive index sensor based on an integrated three- dimensional photonic crystal," Applied Physics Letters, 2008, 92(7): 071108-1-071107-3.

[36] A. P. Zhang, G. Yan, S. Gao, S. He, B. Kim, J. Im, et al., "Microfluidic refractive-index sensors based on small-hole microstructured optical fiber Bragg gratings," Applied Physics Letters, 2011, 98(22): 221109-1-221109-3.

[37] Y. Wang, S. Meng, Y. Liang, L. Li, and W. Peng, "Fiber-optic surface plasmon resonance sensor with multi-alternating metal layers for biological measurement," Photonic Sensors, 2013, 3(3): 202-207.

[38] J. Zhu, L. Qin, S. Song, J. Zhong, and S. Lin, "Design of a surface plasmon resonance sensor based on grating connection," Photonic Sensors, 2015, 5(2): 159-165.

[39] T. J. Huang, M. I. Lapsley, S. C. S. Lin, and X. L. Mao, "An in-plane, variable optical attenuator using a fluid-based tunable reflective interface," Applied Physics Letters, 2009, 95(8): 083507-1-083507-3.

[40] E. Weber and M. J. Vellekoop, "Optofluidic micro-sensors for the determination of liquid concentrations," Lab on a Chip, 2012, 12(19): 3754-3759.

[41] D. Qin, Y. Xia, and G. M. Whitesides, "Soft lithography for micro- and nanoscale patterning," Nature Protocols, 2010, 5(3): 491-502. 